\title{
Skin fibroblasts from individuals with Chediak-Higashi Syndrome (CHS) exhibit hyposensitive immunogenic response
}

Le Wang ${ }^{1 *}$, Kamila Rosamilia Kantovitz ${ }^{1,2+}$, Andrew Robert Cullinane ${ }^{3}$, Francisco Humberto Nociti Jr. ${ }^{1,2}$, Brian Lee Foster ${ }^{1}$, Joseph Concepcion Roney ${ }^{3}$, Anne Bich Tran ${ }^{1}$, Wendy Jewell Introne ${ }^{3}$ and Martha Joan Somerman ${ }^{1}$

\begin{abstract}
Background: Chediak-Higashi Syndrome (CHS) is a rare autosomal recessive disease characterized by immunodeficiency, oculocutaneous albinism, neurological dysfunction, and early death. Individuals with CHS present with increased susceptibility to infections of the skin, upper-respiratory tract, gastrointestinal tract, and oral tissues. Classical CHS is caused by mutations in the gene encoding lysosomal trafficking regulator (LYST). Although defects in cytotoxic T cell lytic secretory granule secretion and neutrophil phagocytosis are suggested to contribute to the immunodeficiency in CHS, the underlying molecular mechanisms are unknown. We hypothesized that skin fibroblasts from CHS subjects exhibit impaired immune response due to defective trafficking of inflammatory factors.
\end{abstract}

Methods and results: Primary skin fibroblasts from CHS subjects or healthy controls were assessed for genes encoding inflammatory response factors using PCR array. At baseline, we found CD14, ILIR1 and TLR-1 were down-regulated significantly ( $\geq 2$ fold change) and the genes encoding $T L R-3, I L-1 \beta$ and IL-6 were up-regulated in CHS cells compared to control cells. When challenged with E. coli lipopolysaccharide (LPS), CHS cells were less responsive than control cells, with only 8 genes significantly up-regulated (3-68 fold change) compared to baseline values, whereas 28 genes in control cells were significantly up-regulated at a much higher magnitude (3-4,629 fold change). In addition, 50\% of the genes significantly up-regulated in LPS-treated control cells were significantly lower in LPS-treated CHS cells. IL-6, a fibroblast-derived proinflammatory cytokine essential for fighting infections was significantly lower in culture media of CHS cells with or without LPS. Furthermore, Western blot and immunofluorescent staining revealed that TLR-2 and TLR-4 were diminished on cell membranes of CHS cells and dissociated from Rab11a.

Conclusions: For the first time, results from our study indicate defective trafficking of TLR-2 and TLR-4 contributes to the hyposensitive response of CHS skin fibroblasts to immunogenic challenge, providing a potential therapeutic target for clinical intervention in CHS.

Keywords: Lysosome trafficking regulator, Intracellular vesicle trafficking, Immunodeficiency, Toll-like receptors

\footnotetext{
* Correspondence: le.wang@nih.gov

${ }^{\dagger}$ Equal contributors

${ }^{1} \mathrm{NIH} / \mathrm{NIAMS}$ - National Institute of Arthritis and Musculoskeletal and Skin

Diseases, Bethesda, MD, USA

Full list of author information is available at the end of the article
} 


\section{Background}

Chediak-Higashi syndrome (CHS; OMIM\# 214500) is a rare autosomal recessive disease characterized by partial oculocutaneous albinism (OCA), immunodeficiency, mild bleeding tendency, and varying neurologic problems $[1,2]$. Among CHS individuals, approximately 15\% develop a milder form of the disease exhibiting an atypical phenotype, while the remaining $85 \%$ develop the more severe classic form of the syndrome at birth or soon after birth. It has been reported that individuals with classical CHS present persistent and recurrent infections in skin, upper-respiratory tract, gastrointestinal tract, and oral tissues $[3,4]$. Individuals with classical $\mathrm{CHS}$ often develop the "accelerated phase", characterized by lymphoproliferative infiltration of the bone marrow and reticuloendothelial system, that is usually fatal unless treated by immunosuppression and bone marrow transplantation (BMT) $[1,2,5]$.

The hallmark diagnostic feature of CHS is the presence of giant inclusions due to fusion of cytoplasmic granules in many cell types, including hematopoietic cells, renal tubular cells, neurons, melanocytes, and fibroblasts $[1,6]$. At the molecular level, the disease is caused by mutations in the lysosomal trafficking regulator gene (LYST, also known as CHS1) on 1q42.1-q42.2, identified after the murine homologue gene beige was discovered [7]. Studies suggest a role for LYST in vesicle formation and transport of proteins, though its dysfunction in the context of CHS is not completely understood $[1,2]$. Results from studies led to the suggestion that the enlarged lysosomes found in CHS cells result from abnormalities in membrane fusion [8] or fission [9], which could occur during the biogenesis of the lysosomes.

The deficiency in intracellular transport of vesicles leads to a generalized immunodeficiency in mice and humans $[10,11]$. Increased susceptibility to infection presented by individuals with CHS is known to be a consequence of impaired secretion of lytic secretory granules by cytotoxic T cells and defective phagocytosis, and chemotaxis by neutrophils $[9,12,13]$. However, other than the professional immune cells, fibroblasts, as active contributors to the regulation of the inflammatory response, provide the first barrier against pathogens [14-16]. As BMT only restores the hematopoietic stem cells but cannot correct the mutation in somatic cells such as skin and gingival fibroblasts, it is important to understand whether LYST dysfunction affects immuneinflammatory functions of fibroblasts.

Toll-like receptors (TLRs) act as essential sensors of pathogen-associated molecular patterns, ranging from lipopeptides to nucleic acids [17]. For example, E. coli lipopolysaccharide (LPS) bound to CD14 and MD-2 is recognized by TLR-4, controlling the expression of genes encoding several inflammatory mediators, including cyclooxygenase-2 (COX-2), and pro-inflammatory cytokines such as interleukin (IL)-1 $\beta$ and -6 [18]. Biological availability of TLRs has been reported to be dependent on lysosomal function, underscoring the importance of a normal lysosomal distribution for a balanced TLR response system [19]. Localization and trafficking of TLRs is essential for pathogen recognition, downstream signaling activation and modulation [19-22].

The aims of this in vitro study were to determine how CHS affects the immune response of skin fibroblasts and to define the mechanisms by which disturbed intracellular trafficking leads to impaired immune responses observed in individuals with CHS. We hypothesized that primary skin fibroblasts obtained from individuals with CHS would exhibit a hyposensitive response to immunogenic challenge.

\section{Methods}

Cell isolation, culture and treatment

A total of three subjects with classic CHS were enrolled in the Institutional Review Board approval (NIH/NHGRI - protocol \#00-HG-0153) study (Table 1). Primary skin fibroblasts were obtained from these individuals with CHS. Briefly, a forearm skin biopsy was obtained under local anesthesia and enzymatically digested with $0.25 \%$ trypsin-EDTA solution (Invitrogen, CA, USA) for 1 hour at $37^{\circ} \mathrm{C}$. Cells were maintained in Dulbecco's modified Eagle medium (DMEM) with 10\% fetal bovine serum (FBS), 1\% L-glutamine, and 1\% penicillin/streptomycin (Gibco BRL) and incubated at $37^{\circ} \mathrm{C}$ in a $5 \% \mathrm{CO}_{2}$ atmosphere. For the control group, cells were purchased from American Type Culture Collection (VA, USA), and stored in liquid nitrogen until use. Cells from passages 2 to 8 were used for all experiments. Twenty-four hours after plating, growth medium was changed to DMEM with 5\% FBS (and penicillin, streptomycin, and L-glutamine). For baseline data, cells were cultured without E.coli LPS and for LPS challenge assay, cells were cultured and treated with LPS at $10 \mathrm{ng} / \mathrm{mL}$ for $3 \mathrm{hrs}$.

\section{Gene expression analysis}

For gene expression analysis, total RNA was obtained from cells in vitro using the RNeasy Micro kit (Qiagen, CA, USA), cDNA was synthesized by using the $\mathrm{RT}^{2}$ First Strand Kit (Qiagen), and samples were analyzed for expression of 84 genes involved in immune-inflammatory regulation by a PCR array platform (PAHS-077Z, SABioscience/ Qiagen). PCR array reactions were performed with the LightCycler 480 system (Roche Diagnostics, IN, USA) following the manufacturer's recommendations. Real time quantitative PCR (RT-qPCR) was performed as described before [23]. Primer sequences used in RT-qPCR are shown in Table 2. 
Table 1 Genotypes of the CHS patients

\begin{tabular}{llllll}
\hline Patients & Phenotype & Genotype & & Mutation type \\
\cline { 3 - 6 } & & Mutation & c.9893delT; p.F3298fsX3304 (exon 43) & Nonsense/Frameshift \\
\hline CHS 4 & Classic & c.1540C > T; p.R514X (exon 5) & c.4353G > A; p.W1451X (exon12) & Frameshift/Nonsense \\
CHS 13 & Classic & c.4322_4325delAGAG;p.E1441VfsX11 (exon 12) & c.10883dupA; p.Y3628X (exon 49) & Nonsense/Nonsense \\
CHS 21 & Classic & c.10883dupA; p.Y3628X (exon 49) & .
\end{tabular}

\section{SDS-PAGE and Western blotting}

For protein analysis, total cellular proteins were extracted from cells using a lysis and extraction kit (Thermo Scientific, MA, USA) following the manufacturer's recommendations. EDTA-free Halt $^{\mathrm{Tm}}$ protease inhibitor cocktail (Thermo Scientific) was included to prevent protein degradation during the extraction process. Protein concentration was determined by the Bradford colorimetric assay (Thermo Scientific).

SDS-PAGE and Western blotting were performed as described previously [23]. Primary antibodies against TLR2 (Abcam, MA, USA), TLR-4 (Abcam), GAPDH (Abcam), and IRDye employed with secondary antibodies (LI-COR Biosciences, NE, USA) were used to detect proteins of interest. Detection was performed using a digital imaging system (ODYSSEY CLx-LI-COR), and digital files were analyzed with Image Studio Software (LI-COR).

\section{Immunofluorescence and membrane staining}

Skin fibroblasts from both control and CHS subjects were grown on 4-well chamber slides for $24 \mathrm{hrs}$, then fixed with $4 \%$ paraformaldehyde (PFA), permeabilized using $0.1 \%$ triton-X-100, and incubated overnight at $4^{\circ} \mathrm{C}$ with rabbit anti-TLR-2 (Abcam), mouse anti-TLR-4 (Abcam), mouse anti-Rab11a (BD Biosciences, CA, USA), and rabbit anti-Rab11a antibodies (Cell Signaling Technology, MA, USA). Donkey anti-mouse or anti-rabbit Alexafluor-488 or -555 conjugated antibodies (Invitrogen, CA, USA) were used as secondary antibodies. The slides were mounted in ProLong Gold anti-fade reagent with DAPI (Invitrogen) and imaged using a Zeiss 510 META confocal laser-scanning microscope with the pinhole set to 1 Airy unit (Carl Zeiss, NY, USA). A series of optical sections were collected from the $x y$ plane and merged into maximum projection images. For membrane staining of TLR-2 and TLR-4, cells were seeded as above and allowed to attach for $24 \mathrm{hrs}$, then cooled to $4^{\circ} \mathrm{C}$ and incubated for 1 hour with rabbit polyclonal antibodies against TLR-2 or mouse monoclonal antibodies against TLR-4 diluted in DMEM. Cells were washed twice for 5 minutes with ice cold PBS to remove unbound antibody, and fixed using $4 \%$ PFA for an additional 30 minutes at $4^{\circ} \mathrm{C}$, and for a further 30 minutes at room temperature. The slides were then processed for immunofluorescence staining as above, using donkey anti-mouse or anti-rabbit Alexafluor-488 conjugated secondary antibodies (Invitrogen). Slides were mounted and imaged as above.

\section{Statistical analysis}

Experiments were performed in triplicate and repeated at least twice. Values are given as means and standard deviations, or as fold-change. An integrated web-based software $\mathrm{RT}^{2}$ Profiler PCR array software package (SABiosciences) was used for PCR array data analysis. In general, the normality of the data is analyzed and the $\mathrm{p}$ values are calculated based on a Student's $t$-test of the replicate $2^{(-\Delta \mathrm{Ct})}$ values for each gene in the control group and treatment groups. Student's $t$-test $(\alpha=0.05)$ was performed for analyzing RT-qPCR data. One-way ANOVA with Tukey's post hoc test were used for quantitation of Western blot and ELISA. Correlation analysis was performed by Pearson product moment correlation co-efficient analyses with two-tailed 95\% confidence. Statistical analyses were carried out using GraphPad Prism software version 6 (GraphPad Software, CA, USA) and RT2 Profiler PCR array software package (SABiosciences).

Table 2 Real-time PCR primer sequences

\begin{tabular}{llll}
\hline Gene & Accession & Prime forward & Prime reverse \\
\hline IL-1 $\beta$ & NM_000576.2 & TACCTGTCCTGCGTGTGAA & TCTTTGGGTAATTTTGGGATCT \\
IL-6 & NM_000600.3 & GATGAGTACAAAAGTCCTGATCCA & CTGCAGCCACTGGTTCTGT \\
COX-2 & NM_000963.2 & CTTCACGCATCAGTTITTCAAG & TCACCGTAAATATGATTTAAGTCCAC \\
TLR-2 & NM_003264.3 & CGTTCTCTCAGGTGACTGCTC & CCTTGGATCCTGCTTGC \\
TLR-3 & NM_003265.2 & AGAGTTGTCATCGAATCAAATTAAAG & AATCTTCCAATTGCGTAAAA \\
TLR-4 & NM_138557.2 & CCTGCGTGAGACCAGAAAG & TTCAGCTCCATGCATTGATAA \\
GAPDH & NM_002046.4 & AGCCACATCGCTCAGACAC & GCCCAATACGACCAAATCC \\
\hline
\end{tabular}




\section{Results}

\section{CHS skin fibroblasts in vitro exhibit hyperactive immune} activity at baseline

To determine how CHS affects expression of genes associated with inflammation and immune response, expression profiles of skin fibroblasts at baseline were screened by PCR array and RT-qPCR. PCR array revealed that CD14, IL-1R1 and TLR-1 were significantly $(\mathrm{p}<0.05)$ down-regulated, with more than a 2 -fold change in $\mathrm{CHS}$ cells compared to control cells (Figure 1A, Table 3). Furthermore, PCR array identified decreased TLR-2 and -4 expression (8- and 4-fold, respectively), and 4fold increased TLR-3 expression in CHS skin fibroblasts compared to control cells, although without significance (Figure 1A, Table 3). RT-qPCR confirmed statistically significant 4-fold down regulation of TLR-4 in CHS cells vs. controls (Figure $1 \mathrm{~B}$ ), while also reconfirming the expression pattern for TLR-2 (3-fold decrease) and TLR-3 (2-fold increase) (Figure 1B and C).

RT-qPCR analysis revealed that in CHS fibroblasts, $I L-6$ was significantly up-regulated (14-fold), and $I L-1 \beta$ was up-regulated (15-fold) with a p-value close to 0.05 (Figure 1D). Furthermore, expression of cyclooxygenase $2(C O X-2)$, an enzyme largely responsible for inducing inflammation, was significantly $(\mathrm{p}<0.01)$ elevated $(16$-fold) in CHS skin fibroblasts compared to control cells. Correlation analysis shown in Table 4 indicated that expression of $C O X-2, I L-6$, and $I L-1 \beta$ were significantly and positively correlated with one another as well as with TLR-3 gene expression, while significantly and negatively correlated with TLR-4 gene expression. Notably, TLR-2 gene expression was not significantly correlated with any of the genes included in Table 4.

\section{CHS skin fibroblasts exhibit a hyposensitive immune response when challenged with LPS}

Next we examined how mutations in LYST affect inflammation and immune response genes of interest (identified in the preceding section and Figure 1) in skin fibroblasts challenged with E. coli LPS. The PCR array revealed that compared to LPS-treated control cells, 14 genes in CHS cells were either decreased significantly or in a declining trend (Figure 2A green circles and Table 5). This global trend indicated that, under LPS challenge, the immune response was repressed in skin fibroblasts of individuals with CHS. Notably, challenged CHS cells exhibited 30-fold lower and 4-fold lower expression of $T L R-2$ and -4 , respectively, compared to challenged control cells (Table 5). Only one gene (LY98) encoding $M D$-2, the LPS recognition partner of TLR-4, was significantly enhanced (2.5-fold) in CHS cells challenged by LPS (Figure $2 \mathrm{~A}$ and Table 5 ).

The observation that $\mathrm{CHS}$ cells exhibited an overall lower level of immunogenic expression when compared

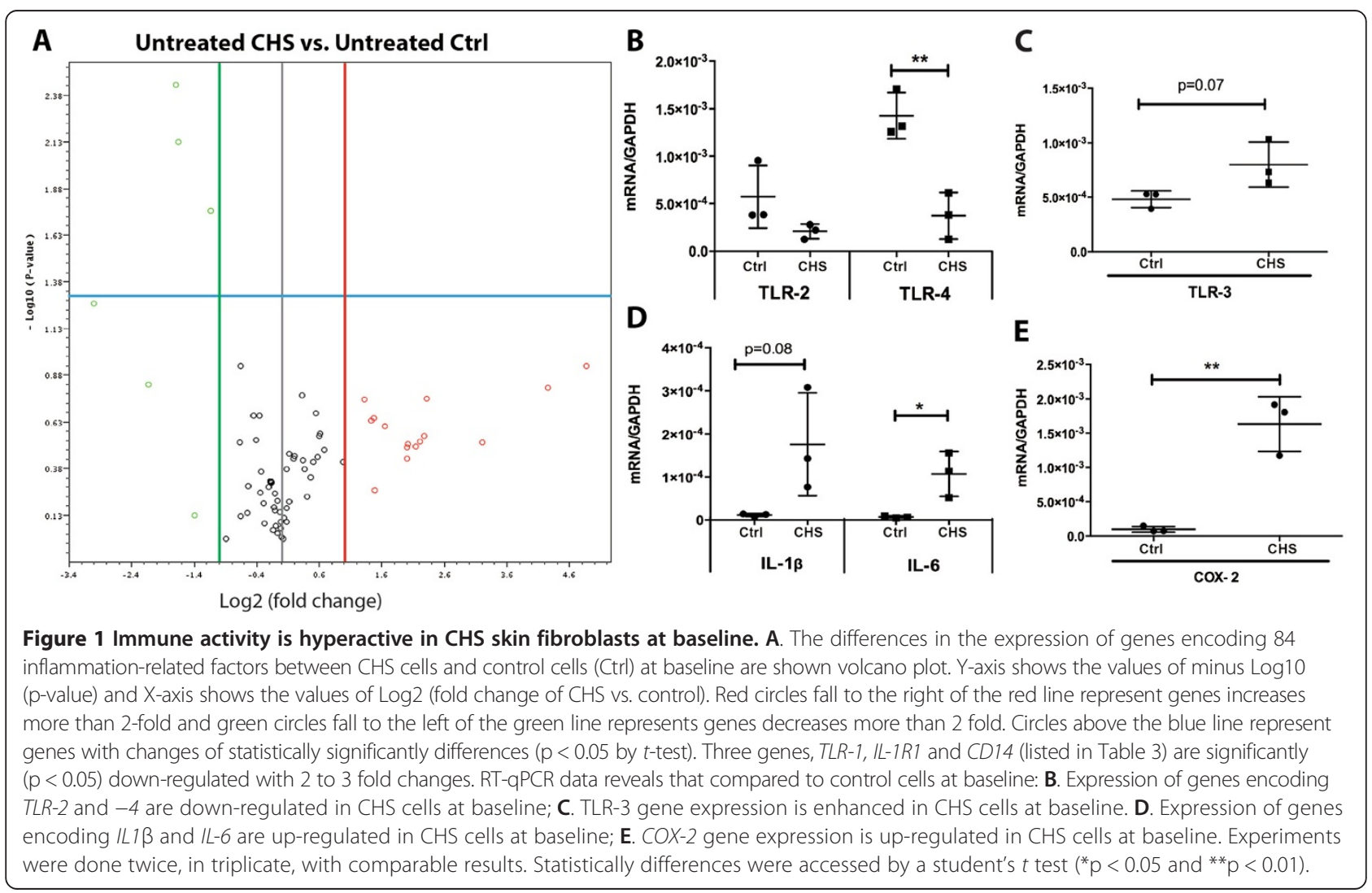


Table 3 Gene expression profile of untreated CHS cells compared to untreated control cells

\begin{tabular}{llcl}
\hline Gene symbol & Gene full name & Fold regulation & p-value \\
\hline TLR1 & Toll-like receptor 1 & -3.25 & 0.00 \\
CD14 & CD14 molecule & -3.14 & 0.01 \\
IL1R1 & Interleukin 1 receptor, type I & -2.19 & 0.02 \\
TLR2 & Toll-like receptor 2 & -8.04 & 0.06 \\
TLR4 & Toll-like receptor 4 & -4.37 & 0.15 \\
IL-6 & Interleukin 6 & 2.50 & 0.18 \\
IL-1 $\beta$ & Interleukin 1 3 & 9.21 & 0.30 \\
TLR-3 & Toll-like receptor 3 & 4.03 & 0.31 \\
\hline
\end{tabular}

Note: Table 3 corresponds to the volcano plot of Figure 1A. Genes above the dotted line have altered expression in CHS cells with statistical significance ( $p<0.05$ ); Genes below the dotted line were evaluated by RT-qPCR.

to control cells treated by LPS does not mean that CHS cells decreased the expression of those specific factors, as shown in Table 5. By comparing stimulated cells to unstimulated cells, we revealed that both $\mathrm{CHS}$ and control cells exhibited increased expression of those specific factors in response to LPS challenge, but CHS cells exhibited a lower fold-response. As shown in Figure 2, only 8 genes were significantly up regulated more than 2-fold (ranging from 2.45- to 75.76-fold) in LPS-treated CHS cells compared to untreated CHS cells (Figure 2B and Table 6). In contrast, LPS-treated control cells exhibit significant up-regulation of 28 genes, more than 2-fold (ranging from 3- to 2,048-fold) compared to untreated control cells (Figure 2C and Table 7). Notably, LPS treatment significantly enhanced expression of TLR2 and -4 in control cells, but not in CHS cells. Consistent with trends in the PCR array data in Figure 2A, LPS-treated CHS cells featured significantly lower magnitude of $I L-1 \beta, I L-6$ and $C O X-2$ induction (40-200 fold) compared to LPS-treated control cells (Figure 2D).

IL-6 is a proinflammatory cytokine that is secreted by fibroblasts [24]. Secreted IL-6 protein in tissue culture media was measured in LPS-treated and untreated cells. The concentration of IL- 6 was significantly lower in medium from CHS cells compared to control cells (Figure 2E). Additionally, exposure to LPS failed to induce increased IL-6 secretion in CHS cells, while LPS-treated control cells exhibited a significant 4-fold increase in IL-6. The contradictory observation of increased IL-6 gene expression and the decreased secretion of IL-6 protein in cell culture of CHS skin fibroblasts suggests a defect in IL-6 secretion is related to LYST mutations. Notably, the difference in secreted IL-6 expression between controls and CHS fibroblasts could partly arise from the source and culturing methods of control and CHS cells. However, both control and CHS cells were cultured under identical conditions in-house and passaged several times before use in experiments, nullifying any a priori differences.

\section{CHS skin fibroblasts exhibit diminished protein expression of TLR-2 and -4}

Toll-like receptors are recognized as playing key roles in innate immune response to immunogenic challenges, such as microbial products including LPS [17]. Lower gene expression of TLR-2 and TLR-4 in CHS fibroblasts compared to controls cells (Figure $1 \mathrm{~A}$ and $\mathrm{B}$ ) prompted us to analyze protein expression and localization to further define the mechanism for the observed defective immune response in CHS cells. At baseline, membranebound TLR-2 and -4 were decreased markedly in CHS versus control cells (Figure $3 \mathrm{~A}-\mathrm{a}, \mathrm{b}$ and $3 \mathrm{~B}-\mathrm{i}, \mathrm{j}$ ), with significant differences confirmed by quantitative analysis of Western blot data (Figure $3 \mathrm{C}$ and $\mathrm{E}$ ).

Cells were permeabilized to visualize cytosolic TLR-2 and -4 , revealing that compared to robust cytosolic TLR-2 in control fibroblasts, cytosolic TLR-2 was not detectable in CHS cells (Figure 3A-f vs. c). Quantitative analysis of Western blot confirmed this observation (Figure 3D). In contrast, cytosolic TLR-4 was comparable

Table 4 Summary of correlation between expression of indicated genes

\begin{tabular}{llllll}
\hline Correlation & TLR-3 & COX-2 & IL-6 & IL-1 $\boldsymbol{\beta}$ & TLR-4 \\
\hline TLR-3 & n/a & $r=+0.882 ; p<0.05$ & $r=+0.857 ; p<0.05$ & $r=+0.978 ; p<0.001$ & n.S. \\
COX-2 & $\#$ & $n / a$ & $n . S$. & $r=+0.884 ; p<0.05$ & $r=-0.911 ; p<0.05$ \\
IL-6 & $\#$ & n.s. & $n / a$ & $r=+0.829 ; p<0.05$ & $r=-0.821 ; p<0.05$ \\
IL-1 $\beta$ & $\#$ & $\#$ & $\#$ & $n / a$ & $r=-0.831 ; p<0.05$ \\
\hline
\end{tabular}

Note: 1. TLR-2 gene expression is not significantly correlated with any of the genes shown in the table. 2. Correlation analysis was performed by Pearson product moment correlation co-efficient analyses. Pearson $r$ represents the correlation coefficiency where 1 is total positive correlation, 0 is no correlation, and - 1 is total negative correlation. The significance is indicated by $\mathrm{p}$ value with two-tailed $95 \%$ confidence. n/a: not available; n.s.: not significant. \#: redundant. 


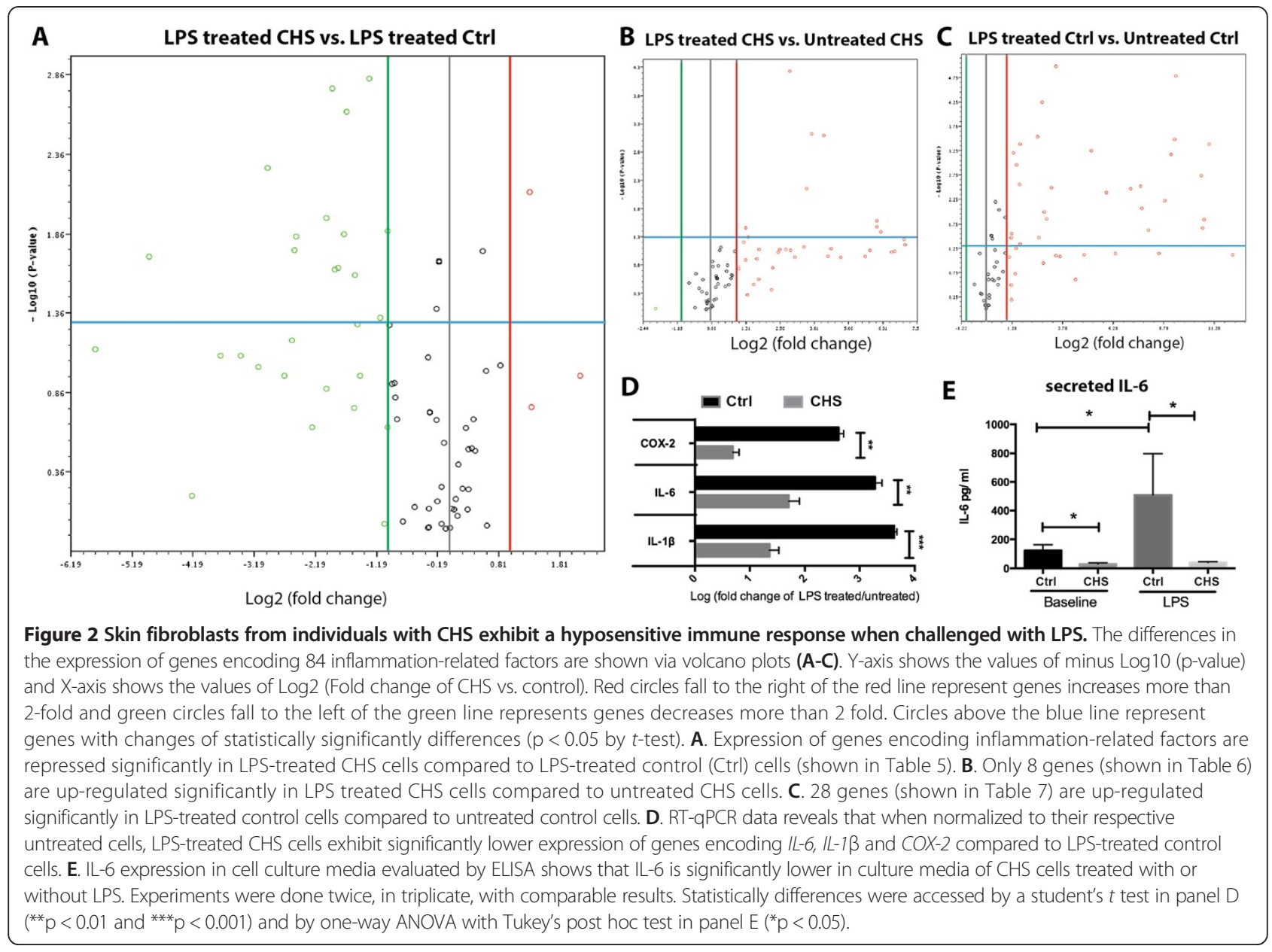

Table 5 Gene expression profile of LPS-treated CHS cells compared to LPS-treated control cells

\begin{tabular}{|c|c|c|c|}
\hline Gene symbol & Gene full name & Fold regulation & p-value \\
\hline TLR2 & Toll-like receptor 2 & -30.20 & 0.02 \\
\hline IL1A & Interleukin 1, alpha & -7.85 & 0.01 \\
\hline LTB & Lymphotoxin beta (TNF superfamily, member 3) & -5.80 & 0.02 \\
\hline IL23A & Interleukin 23, alpha subunit p19 & -5.67 & 0.01 \\
\hline TLR4 & Toll-like receptor 4 & -4.03 & 0.01 \\
\hline CCL7 & Chemokine (C-C motif) ligand 7 & -3.78 & 0.00 \\
\hline CXCL3 & Chemokine (C-X-C motif) ligand 3 & -3.66 & 0.02 \\
\hline CXCR2 & Chemokine (C-X-C motif) receptor 2 & -3.55 & 0.02 \\
\hline TLR1 & Toll-like receptor 1 & -3.31 & 0.01 \\
\hline IL15 & Interleukin 15 & -3.23 & 0.00 \\
\hline CCR1 & Chemokine (C-C motif) receptor 1 & -2.91 & 0.03 \\
\hline IL18 & Interleukin 18 & -2.49 & 0.00 \\
\hline IL10 & Interleukin 10 & -2.20 & 0.05 \\
\hline IL1R1 & Interleukin 1 receptor, type I & -2.02 & 0.01 \\
\hline LY96* & Lymphocyte antigen 96 (MD-2) & 2.49 & 0.01 \\
\hline
\end{tabular}

Note: Table 5 corresponds to the volcano plot of Figure 2A. ${ }^{*}$ LY96, also known as MD-2, was the only gene significantly up-regulated in LPS treated CHS cells. 
Table 6 Gene expression profile of LPS-treated CHS cells compared to untreated CHS cells

\begin{tabular}{|c|c|c|c|}
\hline Gene symbol & Gene full name & Fold regulation & p-value \\
\hline CXCL2 & Chemokine (C-X-C motif) ligand 2 & 75.76 & 0.04 \\
\hline CXCL1 & $\begin{array}{l}\text { Chemokine (C-X-C motif) ligand } 1 \text { (melanoma growth } \\
\text { stimulating activity, alpha) }\end{array}$ & 68.75 & 0.03 \\
\hline IL8 & Interleukin 8 & 68.59 & 0.03 \\
\hline CCL7 & Chemokine (C-C motif) ligand 7 & 17.88 & 0.00 \\
\hline IL1A & Interleukin 1, alpha & 13.09 & 0.00 \\
\hline CXCL6 & Chemokine (C-X-C motif) ligand 6 & 11.50 & 0.01 \\
\hline CCL4 & Chemokine ( $\mathrm{C}-\mathrm{C}$ motif) ligand 4 & 7.53 & 0.00 \\
\hline C3AR1 & Complement component 3a receptor 1 & 2.45 & 0.03 \\
\hline
\end{tabular}

Note: Table 6 corresponds to the volcano plot in Figure 2B.

Table 7 Gene expression profile of LPS-treated control cells compared to untreated control cells

\begin{tabular}{|c|c|c|c|}
\hline Gene symbol & Gene full name & Fold regulation & p-value \\
\hline $\mathrm{CXCL3}$ & Chemokine (C-X-C motif) ligand 3 & 2048.00 & 0.00 \\
\hline CCL5 & Chemokine ( $\mathrm{C}-\mathrm{C}$ motif) ligand 5 & 1722.16 & 0.01 \\
\hline TNF & Tumor necrosis factor & 1652.00 & 0.02 \\
\hline IL1B & Interleukin 1, beta & 1573.76 & 0.00 \\
\hline IL8 & Interleukin 8 & 658.63 & 0.00 \\
\hline $\mathrm{CXCL2}$ & Chemokine (C-X-C motif) ligand 2 & 633.27 & 0.00 \\
\hline CXCL1 & $\begin{array}{l}\text { Chemokine (C-X-C motif) ligand } 1 \\
\text { (melanoma growth stimulating activity, alpha) }\end{array}$ & 557.70 & 0.00 \\
\hline IL6 & Interleukin 6 (interferon, beta 2) & 450.90 & 0.01 \\
\hline SELE & Selectin E & 257.78 & 0.02 \\
\hline PTGS2 & $\begin{array}{l}\text { Prostaglandin-endoperoxide synthase } 2 \\
\text { (prostaglandin } \mathrm{G} / \mathrm{H} \text { synthase and cyclooxygenase) }\end{array}$ & 206.98 & 0.01 \\
\hline CCL2 & Chemokine (C-C motif) ligand 2 & 197.18 & 0.00 \\
\hline IL1A & Interleukin 1, alpha & 141.04 & 0.00 \\
\hline IL23A & Interleukin 23, alpha subunit p19 & 61.53 & 0.00 \\
\hline CCL7 & Chemokine (C-C motif) ligand 7 & 36.50 & 0.00 \\
\hline RIPK2 & Receptor-interacting serine-threonine kinase 2 & 10.78 & 0.00 \\
\hline NFKB1 & Nuclear factor of kappa light polypeptide gene enhancer in B-cells 1 & 9.32 & 0.00 \\
\hline LTB & Lymphotoxin beta (TNF superfamily, member 3) & 7.82 & 0.01 \\
\hline CCL13 & Chemokine (C-C motif) ligand 13 & 7.26 & 0.01 \\
\hline IL15 & Interleukin 15 & 6.74 & 0.00 \\
\hline TLR2 & Toll-like receptor 2 & 6.02 & 0.03 \\
\hline CSF1 & Colony stimulating factor 1 (macrophage) & 5.76 & 0.00 \\
\hline TLR7 & Toll-like receptor 7 & 3.20 & 0.05 \\
\hline IL10 & Interleukin 10 & 3.20 & 0.00 \\
\hline BCL6 & B-cell CLL/lymphoma 6 & 3.13 & 0.00 \\
\hline TLR4 & Toll-like receptor 4 & 2.81 & 0.00 \\
\hline CD40 & CD40 molecule, TNF receptor superfamily member 5 & 2.53 & 0.00 \\
\hline IL18 & Interleukin 1 & 2.39 & 0.03 \\
\hline TLR3 & Toll-like receptor 3 & 2.36 & 0.03 \\
\hline
\end{tabular}

Note: Table 7 corresponds to the volcano plot of Figure $2 \mathrm{C}$. 


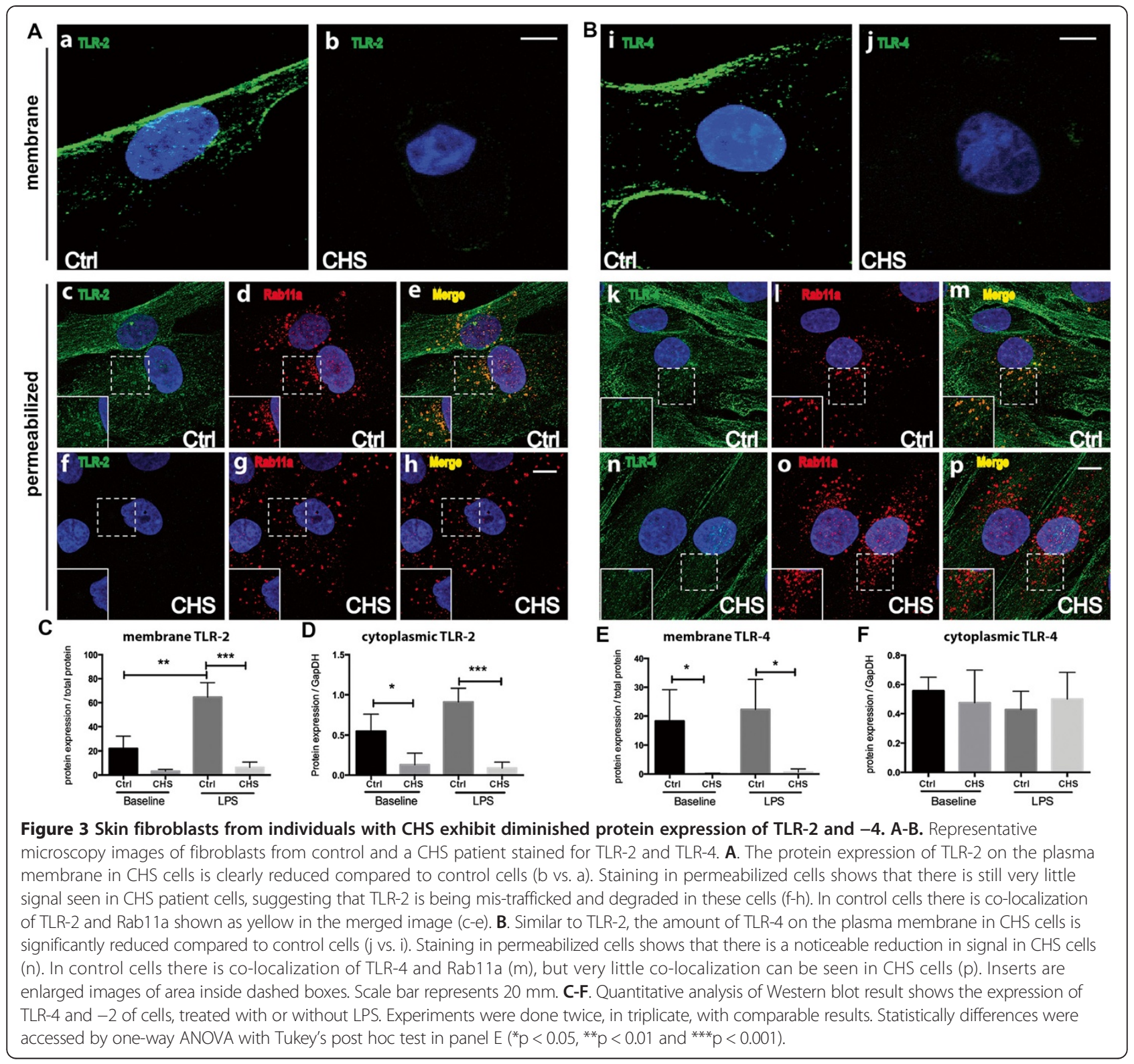

in CHS versus control cells (Figure 3B-n vs. k), and Western blot analysis confirmed this observation (Figure 3F). The Rab11a GTPase has been shown to be involved in the recycling and trafficking of TLRs [20]; therefore we co-stained Rab11a with TLR-2 and -4. In control fibroblasts, Rab11a co-localized with TLR-2 and -4 (Figure 3, A-e and B-m). However, in CHS cells, Rab11a localization was notable for a lack of co-localization with TLR-4 (Figure 3B-p). Furthermore, Western blot revealed that when challenged by LPS, CHS cells exhibited significantly lower expression of TLR-2 compared to control cells (Figure 3C and D). Although intracellular TLR-4 expression in CHS cells was similar to that of control cells, the expression of membrane bound TLR-4 was significantly decreased in CHS cells (Figure 3E and F).

\section{Discussion}

Individuals with CHS are reported to exhibit increased susceptibility to infections in tissues that act as barriers to pathogenic invasion, such as the skin and gingiva $[3,4]$. Fibroblasts, the major cells residing in skin and gingiva, are immune competent cells that participate in signaling and triggering an innate immunity reaction in response to pathogens $[24,25]$. While CHS-induced defects have been studied in major immune cell classes, including neutrophils [26,27], macrophages [28], and T cells [29], little is known about how mutations causing $\mathrm{CHS}$ affect immunogenic responsiveness of fibroblasts. In these in vitro studies of skin fibroblasts obtained from individuals with CHS, we demonstrate hyperactive inflammatory immune activity at baseline, yet hyporesponsiveness to 
immunogenic LPS challenge, potentially due to the reduced expression and disturbed trafficking of TLR-2 and -4 .

Our results demonstrate that at baseline (i.e. unstimulated), $\mathrm{CHS}$ fibroblasts present hyperactive expression of immune-related factors including $I L-1 \beta, I L-6$, and $C O X-2$, compared to cells obtained from normal subjects. Enhanced expression of these factors was significantly and positively correlated with one another and with enhanced TLR-3 expression in CHS cells, suggesting co-regulation by common upstream signals. In fact, TLR-3 is one of the upstream regulators that has been shown to induce expression of the elevated immunerelated factors mentioned above (Figure 1D) [30]. Although $T L R-1,-2,-4$, and CD14 were all decreased in CHS cells, increased expression of TLR-3 may lead to the mild hyperactive immune activity. The marked functional difference between TLR-2 or -4 , and TLR-3, is that TLR-2 and -4 localize to the cell surface in order to recognize foreign lipid structures, and rely on intracellular trafficking to signal and replenish the surface pool of TLR proteins [21], while TLR-3 resides intracellularly in order to recognize nucleic acids delivered by a cytoplasmic lipid raft protein, reftline, and depends on intracellular trafficking differently from TLR-2 and -4 [31,32]. LYST protein, as an intracellular trafficking regulator whose function is reduced in individuals with CHS, may have a more potent effect on highly regulated cell surface expressed TLRs (e.g. TLR-2 and -4), than intracellular and constitutive TLRs (e.g. TLR-3). It is plausible to propose that skin fibroblasts constantly exposed to exogenous viruses exhibit enhanced expression of TLR-3 and its downstream signaling, which is affected differently by LYST mutation compared to cell surface TLRs and in turn leads to a hyperactive immune system at baseline.

In contrast to the hyperactive expression at baseline, skin fibroblasts from CHS individuals exhibit marked hyporesponsiveness to LPS challenge, failing to alter production of chemokines and cytokines including $C C L-7, I L-10, I L-15, I L-18, I L-1 A$, and $I L-23 A$. Repeated activation of TLR signaling results in a reduction in the subsequent proinflammatory cytokine response, a phenomenon known as TLR tolerance [33], as well as changes in expression of other TLRs, known as cross-tolerance [34]. In the case of CHS cells, gene expression and correlation data suggest that constant activation TLR-3 signaling potentially leads to elevated expression of cytokines and chemokines, which in turn represses the expression of TLR-4 and results in hyposensitive immunogenic response. However, it remains unclear why changes in TLR-2 did not correlate with increased expression of other factors. TLR2 signaling induction relies on heterodimerization with TLR-1 or TLR-6 [35-37]. Significantly diminished expression of
TLR-1 in CHS cells may, explain in part, the complexity of the altered TLR-2 expression.

Cytoplasmic membrane-associated TLRs signal through two primary pathways, defined by the adaptor molecules used to initiate each signal cascade. The classical MyD88dependent pathway relies on functioning cell surface TLRs, is common to both TLR-2 and -4 , and leads to rapid activation of transcription factor $\kappa B(N F-\kappa B)$ to induce proinflammatory mediators such as TNF- $\alpha$, IL- 6 and COX-2 [17]. Our results support severely deficient MyD88-dependent TLR signaling in CHS cells, based on the observations of (1) decreased gene expression of TLR-2, -4, and CD14; (2) attenuated cell surface expression of TLR-2 and -4; and (3) diminished response under LPS challenge of MyD88-dependent proinflammatory mediators, e.g. IL-6 and CXCL2. Furthermore, the presence of TLR-4 receptor complexes on the cytoplasmic membrane is maintained via continuous replenishment of TLR-4 from intracellular compartments including the Golgi apparatus and endosomes, which is a process that is governed by small GTPase and MD-2 [22,38]. CHS skin fibroblasts exhibited enhanced expression of $M D$-2, suggesting a compensatory mechanism that minimizes alterations in cytoplasmic TLR- 4 protein in the face of reduced TLR-4 expression. It will be important to investigate how LYST affects interactions between TLR4 and MD-2, in order to modulate cell surface localization of TLR-4.

In addition to MyD88-dependent TLR signaling from the cell membrane, internalization of TLRs can facilitate a second signaling pathway that employs a distinct set of sorting-signaling adaptors, called the MyD88-independent pathway [17]. Although TLR-2 and -4 share some of the MyD88-independent pathway adaptors, signaling activation of this pathway has different consequences for intracellular trafficking of TLR-2 versus TLR-4. Internalization of TLR-4, governed by the small GTPase, Rab11a, is necessary to induce MyD88-independent signaling [20] while internalization of TLR-2 is not necessary for signaling [39]. This difference may underlie the discrepancy in how LYST mutations affect TLR-2 and TLR-4 in CHS cells. In addition, internalization also results in the TLR recycling through ubiquitination [21]. Since TLR-2 and TLR-4 differ in their mechanism of ubiquitination $[40,41]$, incorrect trafficking may lead to severe depletion of TLR-2, but not TLR-4, in CHS cells. Future studies elucidating this mechanism will provide insight into how LYST contributes to TLR-2 and -4 functions and recycling.

To the best of our knowledge, the results reported here demonstrate for the first time the altered expression and localization of TLRs in cells obtained from CHS subjects, and emphasize the importance for understanding the mechanism by which lysosome recycling 
regulates TLR-mediated inflammatory signaling, and immune function in a broader sense. Clinical features resulting from LYST mutations in CHS have much in common with immunodeficiencies caused by TLR signaling defects, such as conditions caused by autosomal recessive mutations in TLR adapters, IRAK-4 and MyD88 (OMIM\# 610799, 607676, 612260). Like mutations in LYST, reduced function of IRAK-4 and MyD88 results in selective impairment of cell responsiveness to TLRs other than TLR-3 [42], and limited presence of IL-6 protein when exposed to TLR agonists [43]. These conditions feature noninvasive pyogenic bacterial infections affecting skin and upper respiratory tract, with occasional periodontal disease [43]. However, patients with MyD-88 and IRAK-4 deficiency show no impaired defense against viral infections [43], due to their normal functional natural killer cells [43] as well as their retained ability to signal through TLR-3/-7/-9 and other non-TLR viral receptors [44]. In contrast, patients with the classical CHS phenotype develop life-threatening haemophagocytic lymphohisticytosis following infections with viruses [5], which may result from dysfunctional natural killer cells lacking cytotoxic activities $[45,46]$ as well as defective trafficking in TLR-3/-7/-9 signaling.

\section{Conclusions}

Taken together, these findings underscore that intracellular vesicle trafficking is essential for normal immune function. Loss of expression or proper localization of TLR-2 and -4, together with the lack of response of cell production of pro-inflammatory cytokines, leads to exacerbated bacterial burden and delayed clearance. A better understanding of mechanisms governing local inflammatory mechanisms may inform strategies for the management of skin lesions burdened by excessive inflammation, in $\mathrm{CHS}$ and other conditions of immunodeficiency.

\footnotetext{
Abbreviations

BCL6: B-cell CLL/lymphoma 6; BMT: Bone marrow transplant; C3AR1: Complement component 3a receptor 1; CCL: Chemokine (C-C motif) ligand; CCR: Chemokine (C-C motif) receptor; CD14: Cluster of differentiation 14; CEBPB: CCAAT/enhancer binding protein (C/EBP), beta; CHS: Chediak-Higashi syndrome; Ctrl: Control; COX-2: Cyclooxygenase-2; CSF1: Colony stimulating factor 1 (macrophage) $\mathrm{CXCL}$, Chemokine (C-X-C motif) ligand; CXCR: Chemokine (C-X-C motif) receptor; DMEM: Dulbecco modified Eagle medium; FBS: Fetal bovine serum; IL: Interleukin; IRAK: Interleukin-1 receptor-associated kinase; LPS: Lipopolysaccharide; LTB: Lymphotoxin beta (TNF superfamily, member 3); LY96: Lymphocyte antigen 96, also known as MD-2; LYST: Lysosomal trafficking regulator; MD-2: Myeloid differentiation factor-2; MyD-88: Myeloid differentiation primary response gene 88; NFKB1: Nuclear factor of kappa light polypeptide gene enhancer in B-cells 1; OCA: Oculocutaneous albinism; PFA: Paraformaldehyde; Rab: Ras superfamily of monomeric G proteins; RIPK2: Receptor-interacting serine-threonine kinase 2; SDS-PAGE: Sodium dodecyl sulfate-Polyacrylamide gel electrophoresis; SELE: Selectin E; TLR: Toll-like receptor; TNF: Tumor necrosis factor.
}

\section{Competing interests}

The authors declare that they have no competing interests.

\section{Authors' contributions}

LW and KRK co-drafted the manuscript. LW, KRK, FHN and ABT carried out the clinical work and performed the gene expression analysis. FHN and LW carried out protein extraction, Western blots and ELISA. ARC and JCR designed and performed the immunofluorescence and membrane staining. BLF, MJS and WJ provided advice on experimental design, and critically read and revised the manuscript. MJS and WJI contributed clinical background and supervised the study. LW, KRK, FHN and ARC participated in the design of the study and performed the statistical analysis. All authors read and approved the final manuscript.

\section{Acknowledgments}

This research was supported by the Intramural Research Program of NIAMS (LW, KRK, FHN, BLF, ABT, MJS) and NHGRI (ARC, JCR and WJI) of the NIH. The authors acknowledge Dr. Samuel Black (University of Massachusetts at Amherst), Dr. Richard Darveau (University of Washington), and Dr. Vivek Thumbigere-Math (NIAMS/NIH, Bethesda, MD) for scientific advice, and Mudita Patel (NIAMS/NIH, Bethesda, MD) for technique assistance.

\section{Author details}

${ }^{1} \mathrm{NIH} / \mathrm{NIAMS}$ - National Institute of Arthritis and Musculoskeletal and Skin Diseases, Bethesda, MD, USA. ${ }^{2}$ University of Campinas - Piracicaba Dental School, Piracicaba, Sao Paulo, Brazil. ${ }^{3} \mathrm{NIH} / \mathrm{NHGRI}$ - National Human Genome Research Institute, Bethesda, MD, USA.

Received: 13 August 2014 Accepted: 10 December 2014

Published online: 21 December 2014

\section{References}

1. Introne W, Boissy RE, Gahl WA: Clinical, molecular, and cell biological aspects of Chediak-Higashi syndrome. Mol Genet Metab 1999, 68(2):283-303.

2. Kaplan J, De Domenico I, Ward DM: Chediak-Higashi syndrome. Curr Opin Hematol 2008, 15(1):22-29.

3. Roy A, Kar R, Basu D, Srivani S, Badhe BA: Clinico-hematological profile of Chediak-Higashi syndrome: experience from a tertiary care center in south India. Indian J Pathol Microbiol 2011, 54(3):547-551.

4. Armitage GC: Development of a classification system for periodontal diseases and conditions. Ann Periodontol 1999, 4(1):1-6.

5. Lozano ML, Rivera J, Sanchez-Guiu I, Vicente V: Towards the targeted management of Chediak-Higashi syndrome. Orphanet J Rare Dis 2014, 9:132.

6. Zhao H, Boissy YL, Abdel-Malek Z, King RA, Nordlund JJ, Boissy RE: On the analysis of the pathophysiology of Chediak-Higashi syndrome. Defects expressed by cultured melanocytes. Lab Invest 1994, 71(1):25-34.

7. Nagle DL, Karim MA, Woolf EA, Holmgren L, Bork P, Misumi DJ, McGrail SH, Dussault BJ Jr, Perou CM, Boissy RE, Duyk GM, Spritz RA, Moore KJ: Identification and mutation analysis of the complete gene for Chediak-Higashi syndrome. Nat Genet 1996, 14(3):307-311.

8. Jones KL, Stewart RM, Fowler M, Fukuda M, Holcombe RF: Chediak-Higashi lymphoblastoid cell lines: granule characteristics and expression of lysosome-associated membrane proteins. Clin Immunol Immunopathol 1992, 65(3):219-226.

9. Durchfort N, Verhoef S, Vaughn MB, Shrestha R, Adam D, Kaplan J, Ward DM: The enlarged lysosomes in beige $\mathrm{j}$ cells result from decreased lysosome fission and not increased lysosome fusion. Traffic 2012, 13(1):108-119.

10. Stossel TP, Root RK, Vaughan M: Phagocytosis in chronic granulomatous disease and the Chediak-Higashi syndrome. N Engl J Med 1972, 286(3):120-123.

11. Wang JW, Howson J, Haller E, Kerr WG: Identification of a novel lipopolysaccharide-inducible gene with key features of both A kinase anchor proteins and chs1/beige proteins. J Immunol 2001, 166(7):4586-4595.

12. Boxer LA, Smolen JE: Neutrophil granule constituents and their release in health and disease. Hematol Oncol Clin North Am 1988, 2(1):101-134.

13. Ganz T, Metcalf JA, Gallin JI, Boxer LA, Lehrer RI: Microbicidal/cytotoxic proteins of neutrophils are deficient in two disorders: Chediak-Higashi syndrome and "specific" granule deficiency. J Clin Invest 1988, 82(2):552-556 
14. Jordana M, Sarnstrand B, Sime PJ, Ramis I: Immune-inflammatory functions of fibroblasts. Eur Respir J 1994, 7(12):2212-2222.

15. Newton K, Dixit VM: Signaling in innate immunity and inflammation. Cold Spring Harbor perspectives in biology 2012, 4(3):a006049.

16. Silzle T, Randolph GJ, Kreutz M, Kunz-Schughart LA: The fibroblast: sentinel cell and local immune modulator in tumor tissue. Int J Cancer 2004, 108(2):173-180.

17. O'Neill LA, Golenbock D, Bowie AG: The history of Toll-like receptors - redefining innate immunity. Nat Rev Immunol 2013, 13(6):453-460

18. Wang PL, Ohura K: Porphyromonas gingivalis lipopolysaccharide signaling in gingival fibroblasts-CD14 and Toll-like receptors. Crit Rev Oral Biol Med 2002 13(2):132-142.

19. Sanjuan MA, Milasta S, Green DR: Toll-like receptor signaling in the lysosomal pathways. Immunol Rev 2009, 227(1):203-220.

20. Husebye H, Aune MH, Stenvik J, Samstad E, Skjeldal F, Halaas O, Nilsen NJ, Stenmark H, Latz E, Lien E, Mollnes TE, Bakke O, Espevik T: The Rab11a GTPase controls Toll-like receptor 4-induced activation of interferon regulatory factor-3 on phagosomes. Immunity 2010, 33(4):583-596.

21. McGettrick AF, O'Neill LA: Localisation and trafficking of Toll-like receptors: an important mode of regulation. Curr Opin Immunol 2010, 22(1):20-27.

22. Wang D, Lou J, Ouyang C, Chen W, Liu Y, Liu X, Cao X, Wang J, Lu L: Ras-related protein Rab10 facilitates TLR4 signaling by promoting replenishment of TLR4 onto the plasma membrane. Proc Natl Acad SC U S A 2010, 107(31):13806-13811.

23. Nociti FH Jr, Foster BL, Tran AB, Dunn D, Presland RB, Wang L, Bhattacharyya $\mathrm{N}$, Collins MT, Somerman MJ: Vitamin D represses dentin matrix protein 1 in cementoblasts and osteocytes. J Dent Res 2014, 93(2):148-154.

24. Chen B, Tsui S, Smith TJ: IL-1 beta induces IL- 6 expression in human orbital fibroblasts: identification of an anatomic-site specific phenotypic attribute relevant to thyroid-associated ophthalmopathy. J Immunol 2005, 175(2):1310-1319.

25. Hatakeyama J, Tamai R, Sugiyama A, Akashi S, Sugawara S, Takada H: Contrasting responses of human gingival and periodontal ligament fibroblasts to bacterial cell-surface components through the CD14/Toll-like receptor system. Oral Microbiol Immunol 2003, 18(1):14-23.

26. Kjeldsen L, Calafat J, Borregaard N: Giant granules of neutrophils in Chediak-Higashi syndrome are derived from azurophil granules but not from specific and gelatinase granules. J Leukoc Biol 1998, 64(1):72-77.

27. White JG, Clawson CC: The Chediak-Higashi syndrome; the nature of the giant neutrophil granules and their interactions with cytoplasm and foreign particulates. I. Progressive enlargement of the massive inclusions in mature neutrophils. II. Manifestations of cytoplasmic injury and sequestration. III. Interactions between giant organelles and foreign particulates. Am J Pathol 1980, 98(1):151-196.

28. Mahoney KH, Morse SS, Morahan PS: Macrophage functions in beige (Chediak-Higashi syndrome) mice. Cancer Res 1980, 40(11):3934-3939.

29. Argyle JC, Kjeldsberg CR, Marty J, Shigeoka AO, Hill HR: T-cell lymphoma and the Chediak-Higashi syndrome. Blood 1982, 60(3):672-676.

30. Stowell NC, Seideman J, Raymond HA, Smalley KA, Lamb RJ, Egenolf DD, Bugelski PJ, Murray LA, Marsters PA, Bunting RA, Flavell RA, Alexopoulou L, San Mateo LR, Griswold DE, Sarisky RT, Mbow ML, Das AM: Long-term activation of TLR3 by poly (I:C) induces inflammation and impairs lung function in mice. Respir Res 2009, 10:43

31. Tatematsu M, Nishikawa F, Seya T, Matsumoto M: Toll-like receptor 3 recognizes incomplete stem structures in single-stranded viral RNA. Nat Commun 2013, 4:1833.

32. Watanabe A, Tatematsu M, Saeki K, Shibata S, Shime H, Yoshimura A Obuse C, Seya T, Matsumoto M: Raftlin is involved in the nucleocapture complex to induce poly(I:C)-mediated TLR3 activation. J Biol Chem 2011, 286(12):10702-10711.

33. Broad A, Kirby JA, Jones DE, Applied I, Transplantation Research G: Toll-like receptor interactions: tolerance of MyD88-dependent cytokines but enhancement of MyD88-independent interferon-beta production. Immunology 2007, 120(1):103-111.

34. Peroval MY, Boyd AC, Young JR, Smith AL: A critical role for MAPK signalling pathways in the transcriptional regulation of toll like receptors. PLoS One 2013, 8(2):e51243.

35. Farhat K, Riekenberg S, Heine H, Debarry J, Lang R, Mages J, Buwitt-Beckmann U, Roschmann K, Jung G, Wiesmuller KH, Ulmer AJ: Heterodimerization of
TLR2 with TLR1 or TLR6 expands the ligand spectrum but does not lead to differential signaling. J Leukoc Bio/ 2008, 83(3):692-701.

36. Jin MS, Kim SE, Heo JY, Lee ME, Kim HM, Paik SG, Lee H, Lee JO: Crystal structure of the TLR1-TLR2 heterodimer induced by binding of a tri-acylated lipopeptide. Cell 2007, 130(6):1071-1082.

37. Takeuchi O, Sato S, Horiuchi T, Hoshino K, Takeda K, Dong Z, Modlin RL, Akira S: Cutting edge: role of Toll-like receptor 1 in mediating immune response to microbial lipoproteins. J Immunol 2002, 169(1):10-14.

38. Calvano JE, Agnese DM, Um JY, Goshima M, Singhal R, Coyle SM, Reddell MT, Kumar A, Calvano SE, Lowry SF: Modulation of the lipopolysaccharide receptor complex (CD14, TLR4, MD-2) and toll-like receptor 2 in systemic inflammatory response syndrome-positive patients with and without infection: relationship to tolerance. Shock 2003, 20(5):415-419.

39. Triantafilou M, Manukyan M, Mackie A, Morath S, Hartung T, Heine H, Triantafilou K: Lipoteichoic acid and toll-like receptor 2 internalization and targeting to the Golgi are lipid raft-dependent. J Biol Chem 2004, 279(39):40882-40889.

40. Chuang $\mathrm{TH}$, Ulevitch RJ: Triad3A, an E3 ubiquitin-protein ligase regulating Toll-like receptors. Nat Immunol 2004, 5(5):495-502.

41. Nakamura M, Watanabe J, Watanabe N: Ubiquitin-like protein MNSFbeta regulates TLR-2-mediated signal transduction. Mol Cell Biochem 2012, 364(1-2):39-43.

42. Picard C, Casanova JL, Puel A: Infectious diseases in patients with IRAK-4 MyD88, NEMO, or IkappaBalpha deficiency. Clin Microbiol Rev 2011, 24(3):490-497.

43. Picard $\mathrm{C}$, von Bernuth $\mathrm{H}$, Ghandil $\mathrm{P}$, Chrabieh $\mathrm{M}$, Levy $\mathrm{O}$, Arkwright $\mathrm{PD}$, McDonald D, Geha RS, Takada H, Krause JC, Creech CB, Ku CL, EhI S, Maródi L, Al-Muhsen S, Al-Hajjar S, Al-Ghonaium A, Day-Good NK, Holland SM, Gallin II, Chapel H, Speert DP, Rodriguez-Gallego C, Colino E, Garty BZ, Roifman C, Hara T, Yoshikawa H, Nonoyama S, Domachowske J, et al: Clinical features and outcome of patients with IRAK-4 and MyD88 deficiency. Medicine 2010, 89(6):403-425.

44. Pennini ME, Perkins DJ, Salazar AM, Lipsky M, Vogel SN: Complete dependence on IRAK4 kinase activity in TLR2, but not TLR4, signaling pathways underlies decreased cytokine production and increased susceptibility to Streptococcus pneumoniae infection in IRAK4 kinase-inactive mice. J Immunol 2013, 190(1):307-316.

45. Nair MP, Gray RH, Boxer LA, Schwartz SA: Deficiency of inducible suppressor cell activity in the Chediak-Higashi syndrome. Am J Hematol 1987, 26(1):55-66.

46. Targan SR, Oseas R: The "lazy" NK cells of Chediak-Higashi syndrome. J Immunol 1983, 130(6):2671-2674.

\section{Submit your next manuscript to BioMed Central and take full advantage of:}

- Convenient online submission

- Thorough peer review

- No space constraints or color figure charges

- Immediate publication on acceptance

- Inclusion in PubMed, CAS, Scopus and Google Scholar

- Research which is freely available for redistribution 